\title{
A Comprehensive Literature Review of the Pelvis and the Lower Extremity FE Human Models under Quasi-static Conditions
}

\author{
Al-Dirini R.M.A. ${ }^{*}$, Thewlis D. ${ }^{a}$, Paul G. ${ }^{a}$ \\ Affiliations: ${ }^{a}$ University of South Australia, Adelaide, Australia
}

\begin{abstract}
Finite Element Modeling (FEM) has become a vital tool in the automotive design and development processes. FEM of the human body is a technique capable of estimating parameters that are difficult to measure in experimental studies with the human body segments being modeled as complex and dynamic entities. Several studies have been dedicated to attain close-to-real FEMs of the human body (Pankoke and Siefert 2007; Amann, Huschenbeth et al. 2009; ESI 2010). The aim of this paper is to identify and appraise the state-of-the art models of the human body which incorporate detailed pelvis and/or lower extremity models. Six databases and search engines were used to obtain literature, and the search was limited to studies published in English since 2000. The initial search results identified 636 pelvis-related papers, 834 buttocks-related papers, 505 thigh-related papers, 927 femur-related papers, 2039 knee-related papers, 655 shank-related papers, 292 tibia-related papers, 110 fibula-related papers, 644 ankle-related papers, and 5660 foot-related papers. A refined search returned 100 pelvis-related papers, 45 buttocks-related papers, 65 thigh-related papers, 162 femur-related papers, 195 kneerelated papers, 37 shank-related papers, 80 tibia-related papers, 30 fibula-related papers and 102 ankle-related papers and 246 foot-related papers. The refined literature list was further restricted by appraisal against a modified LOW appraisal criteria. Studies with unclear methodologies, with a focus on populations with pathology or with sport related dynamic motion modeling were excluded. The final literature list included fifteen models and each was assessed against the percentile the model represents, the gender the model was based on, the human body segment/segments included in the model, the sample size used to develop the model, the source of geometric/anthropometric values used to develop the model, the posture the model represents and the finite element solver used for the model. The results of this literature review provide indication of bias in the available models towards 50th percentile male modeling with a notable concentration on the pelvis, femur and buttocks segments.
\end{abstract}

Keywords: Advanced biomechanical models, Comfort/discomfort, Biomechanics.

\section{Introduction}

Finite Element Modeling (FEM) has become a common tool of increasing importance used to assess the performance of a product or task. Initially, applications of FEM were limited due to the limitations of the processing power available. Rapid improvements in hardware and software combinations have resulted in significant improvements in computational power. FEM of the human body has provided significant insight into the "suitability" of a certain environment or task to the human performing this task. Nowadays, Finite Element Models of the human body are used to assess safety (Vezin and Verriest 2005), comfort (Amann, Klisch et al. 2005; Pankoke and Siefert 2007), and disease processes and treatments (Makhsous and Lin 2009) along with other biomechanical applications.

Standing, sitting and lying postures are the most evident postures in humans' daily activities, however, under the influence of the global tendency towards automation, humans tend to sit for longer times. Thus, understanding the mechanics of the human body while sitting is of interest to many; for example, an automotive seat designer knowing the resonant frequency of the occupant can avoid

*Corresponding author. Email: rami.al-dirini@unisa.edu.au 
designing a seat having the same resonant frequency.

Finite Element Models of humans in a sitting posture date back to the late 1970's (Chow and Odell 1978). Since then, significant research has been conducted to achieve more accurate models of a seated human. Given the mechanical complexity of the human body, researchers modeled the human body into segments and parts. Additionally, the behavior of the human body is highly governed by the environmental conditions imposed upon it, which can be classified into dynamic, static and quasi-static conditions.

The aim of this review is to critically analyze the latest finite element models developed to represent the pelvis and lower extremity of a human body behaving under quasi-static conditions. Findings of this study will help guiding researchers while developing more advanced finite element human models.

\section{Methodology}

\subsection{System Decomposition}

To achieve a reliable search methodology, a system decomposition approach was adopted. In system decomposition, the system is repeatedly divided until a set of less complex sub-systems has been identified to collectively form the whole initial system. According to McConville et al. (McConville, Clauser et al. 1980), the lower part of the human body can be divided into seven segments; pelvis, buttocks, hip, thigh, knee, shank, and foot. Additionally, the ankle joint can be considered as a separate segment. Finally, the femur, the fibula and the tibia can also be considered as independent segments. Consequently, the lower part of the human body can be segmented into eleven separate segments: the pelvis, the buttocks, the hip, the knee, the shank, the ankle, the foot, the femur, the fibula, and the tibia.

\subsection{Search Methodology}

In this review, the following databases were searched for relevant publications:

- Science Direct

- IEEE Xplore

- ISI Web of Knowledge

- PubMed
- Scopus

- Google Scholar

At the first stage, the keywords used were in the form: [Finite Element Model AND \{Lower extremity OR pelvis OR buttocks OR hip OR thigh OR knee OR shank OR tibia OR fibula OR femur OR ankle OR foot\} AND Quasi Static]. The search in each database was filtered to only include related articles written in English and published after 2000. The search returned a total of 12302 search hits, without considering any repetitions amongst the different databases. The search keywords were refined by including "AND Quasi Static" and "AND Digital Human" as well as including a set of quotation marks around the words 'Finite Elements'. The refined search keywords became ["Finite Element" Model AND \{Lower extremity OR pelvis OR buttocks OR hip OR thigh OR knee OR shank OR tibia OR fibula OR femur OR ankle OR foot $\}$ AND Quasi Static AND Digital Human]. The modified search returned a total of 1062 hits, not eliminating repeated search results within different databases. As a final stage of the search, studies with unclear methodologies, with a focus on populations with pathology or with sport-related dynamic motion modeling were excluded. The final literature list was included a total of fifteen studies all of which match the aforementioned inclusion criteria. In this review, the literature list was appraised against six main criteria, the source of modeling data, the percentile and gender of the model, the sample size, the posture of the model, the body segments included in the model, and the finite element solver and mesh properties. 


\subsection{Critical Appraisal Tools}

To quantity the methodological shortcomings of each individual paper a critical appraisal tool was used (modified LOW critical appraisal criteria, The Critical Appraisal Skills Programme - CASP) [Guyatt, Sackett et al. 1993]). The critical appraisal specifies eleven criteria against which each study is screened. The number of questions answered with a "YES" represents the score out of 11. In this review, the CASP screening questions are redesigned to simplify the screening process. The first column of Table 3 represents the eleven questions each study, out of the fifteen selected studies had to be screened against.

\section{Results: Appraisal of the final literature list}

The initial search results were reduced from a total of 12302 search results to 15 studies (Figure 1). Initially, search results were reduced as a result of the refinement of the search keywords. Out of the refined search, 146 repetitions were noticed and eliminated. The titles and/or abstracts of the remaining 893 search hits were reviewed and any irrelevant hits were excluded. As a final stage, the full papers were reviewed and irrelevant studies were excluded, leaving a total of fifteen relevant studies to analyze and inspect. A thorough inspection of these 15 studies would provide a clear understanding of what is being done and what is still not ventured in the FEM of the lower part of the human body.

\subsection{Percentile and gender of the model}

The studies listed in the final literature list for this review showed a variation in the gender and percentile of the model developed during each study. However, out of the fifteen studies six did not specify the gender and the percentile of their model (Camacho, Ledoux et al. 2002; Mattingly, Talwalkar et al. 2006; Martins, Pato et al. 2007; Shim, Pitto et al. 2007; Makhsous and Lin 2009; Tang, Chan et al. 2010), three studies developed subject-specific models based on data obtained from male subjects (Phillips, Pankaj et al. 2007; Wagnac, Aubin et al. 2008; Bai, Wei et al. 2010), two studies developed models to represent a $50^{\text {th }}$ percentile male 


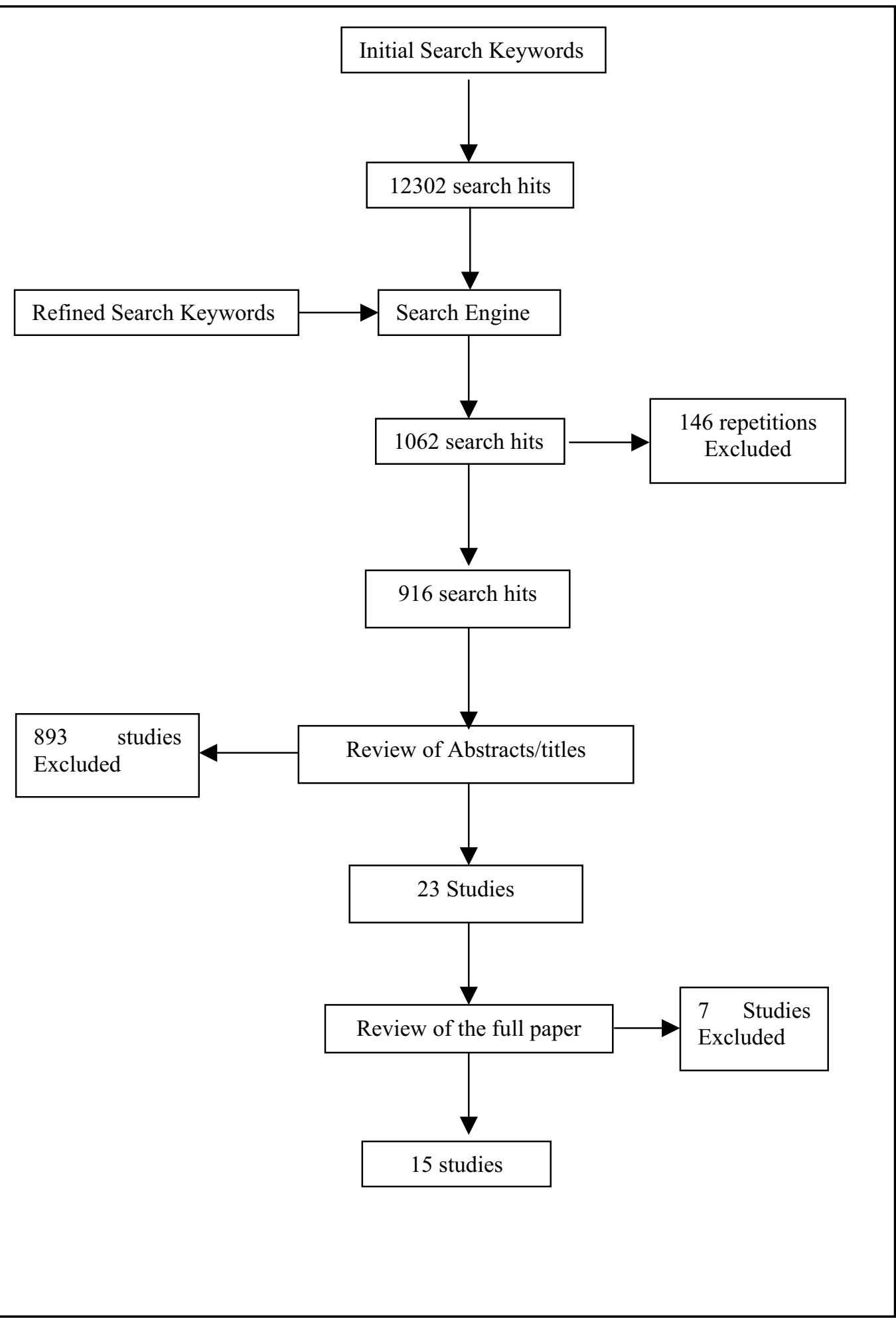

Figure 1: Literature Search Flow Chart 
(Cheng, Smith et al. 2007; Silvestri and Ray 2009) and two developed models to represent three percentiles $\left(5^{\text {th }}\right.$ percentile female, $50^{\text {th }}$ percentile male and $95^{\text {th }}$ percentile male) (Vezin and Verriest 2005; Kim, Pyun et al. 2010).

\subsection{Source of data}

Throughout all of the literature discussed in this review, two main sources of data were noted. The first was through available databases such as the Visible Human Project (VHP 2011), the Chinese Visible Human Project (CVHP 2011), VAKHUM (VAKHUM 2011), and SizeUSA (SizeUSA 2011). Out of the fifteen studies in this review, three studies (Shim, Pitto et al. 2007; Wagnac, Aubin et al. 2008; Silvestri and Ray 2009) obtained their data from the Visible Human project, Whereas Cheng et al. (Cheng, Smith et al. 2007) obtained their data from VAKHUM, Kim et al. (Kim, Pyun et al. 2010) from SizeUSA database and Bai el al. (Bai, Wei et al. 2010) from the Chinese Visible Human project. The second noted source of data was from dedicated in-vivo or in-vitro scans. The literature review identified three studies to have obtained their data from cadavers (Camacho, Ledoux et al. 2002; Anderson, Peters et al. 2004; Martins, Pato et al. 2007) and five studies obtained their data from live subject scans (X-ray scans (Vezin and Verriest 2005), 3D scans (Phillips, Pankaj et al. 2007), MRIs (Mattingly, Talwalkar et al. 2006; Makhsous and Lin 2009), and CT scans (El-Asfoury and El-Hadek 2009)). The remaining study did not specify the source of their data (Tang, Chan et al. 2010).

\subsection{Sample Size}

In three studies, finite element models were developed based on data collected from single subjects (Phillips, Pankaj et al. 2007; Wagnac, Aubin et al. 2008; Bai, Wei et al. 2010), one study modeled based on data collected from three subjects, while Cheng et al. produced their model based on data from five subjects (Cheng, Smith et al. 2007). Makhsous et al. created their model from data collected from eleven subjects (Makhsous and Lin 2009) whereas Mattingly et al. developed their model based on six subjects and sixty four MRIs (Mattingly, Talwalkar et al. 2006). Vezin et al. based their model on data collected from a significantly larger sample size, a total of sixty four subjects of different genders and statures (Vezin and Verriest 2005)The rest of the studies did not specify a sample size for their models.

\subsection{Posture of Model}

Examination of the fifteen studies showed that out of the total fifteen studies, six did not specify a posture for the model (Camacho, Ledoux et al. 2002; Martins, Pato et al. 2007; Shim, Pitto et al. 2007; El-Asfoury and El-Hadek 2009; Silvestri and Ray 2009; Bai, Wei et al. 2010), three models were developed to represent a human in a seated posture (Vezin and Verriest 2005; Cheng, Smith et al. 2007; Tang, Chan et al. 2010), one study specified a nonweighted sitting posture for the subjects while the data was collected (Wagnac, Aubin et al. 2008), one specified a driving posture (Kim, Pyun et al. 2010), while Makhsous et. al (Makhsous and Lin 2009) specified that subjects were sitting with an 80 degree flexion of the hip and a 90 degree flexion of the knee under two different loading conditions, a simulated sitting pressure (in an closed MRI scanner, the pressure was simulated by placing a cushion under the buttock of the subject) and a nositting pressure (in an open MRI scanner, the subject was upright-seated and underarm supports were placed to bear the weight of the subject). On the other hand, Phillips et al. collected data from subjects while standing on one leg (Phillips, Pankaj et al. 2007), and Mattingly et al. (Mattingly, Talwalkar et al. 2006) and Anderson et al. (Anderson, Peters et al. 2004) did not specify a posture for the model as their models only represent the foot and the pelvis respectively, and so regarded posture as a parameter that does not apply to their models.

\subsection{The Body Segment Modeled}

The studies included in this literature review indentified two whole-body models, ten pelvis models, five buttocks models, three hip models, five thigh models, four foot models, three knee models, two ankle models, seven femur models, three fibula models, four tibia models and two shank models. 


\section{Table 1: Body segments Summary}

\begin{tabular}{|l|l|}
\hline Body segment & Studies which modeled the segment \\
\hline Whole body & $\begin{array}{l}\text { 2 Studies } \\
\text { (Bai, Wei et al. 2010; Kim, Pyun et } \\
\text { al. 2010) }\end{array}$ \\
\hline Pelvis & $\begin{array}{l}\text { 10 Studies } \\
\text { (Anderson, Peters et al. 2004; Vezin } \\
\text { and Verriest 2005; Cheng, Smith et } \\
\text { al. 2007; Martins, Pato et al. 2007; } \\
\text { Phillips, Pankaj et al. 2007; Shim, } \\
\text { Pitto et al. 2007; El-Asfoury and El- } \\
\text { Hadek 2009; Silvestri and Ray } \\
\text { 2009; Bai, Wei et al. 2010; Kim, } \\
\text { Pyun et al. 2010) }\end{array}$ \\
\hline Buttocks & $\begin{array}{l}\text { 5 Studies } \\
\text { (Wagnac, Aubin et al. 2008; } \\
\text { Makhsous and Lin 2009; Bai, Wei } \\
\text { et al. 2010; Kim, Pyun et al. 2010; } \\
\text { Tang, Chan et al. 2010) }\end{array}$ \\
\hline Hip & $\begin{array}{l}\text { 3 Studies } \\
\text { (Silvestri and Ray 2009; Bai, Wei et } \\
\text { al. 2010; Kim, Pyun et al. 2010) }\end{array}$ \\
\hline 5 Studies \\
(Wagnac, Aubin et al. 2008; \\
Silvestri and Ray 2009; Bai, Wei et \\
al. 2010; Kim, Pyun et al. 2010; \\
Tang, Chan et al. 2010) \\
2 Studies \\
(Bai, Wei et al. 2010; Kim, Pyun et \\
al. 2010)
\end{tabular}

\begin{tabular}{|l|l|}
\hline Knee & $\begin{array}{l}\text { 3 Studies (Silvestri and Ray 2009; } \\
\text { Bai, Wei et al. 2010; Kim, Pyun et } \\
\text { al. 2010) }\end{array}$ \\
\hline Ankle & $\begin{array}{l}\text { 2 Studies } \\
\text { (Bai, Wei et al. 2010; Kim, Pyun et } \\
\text { al. 2010) }\end{array}$ \\
\hline Femur & $\begin{array}{l}\text { 7 Studies } \\
\text { (Vezin and Verriest 2005; Shim, } \\
\text { Pitto et al. 2007; Silvestri and Ray } \\
\text { 2009; Bai, Wei et al. 2010; Kim, } \\
\text { Pyun et al. 2010; Tang, Chan et al. } \\
\text { 2010) }\end{array}$ \\
\hline Fibula & $\begin{array}{l}\text { 3 Studies } \\
\text { (Vezin and Verriest 2005; Bai, Wei } \\
\text { et al. 2010; Kim, Pyun et al. 2010) }\end{array}$ \\
\hline Tibia & $\begin{array}{l}\text { 4 Studies } \\
\text { (Vezin and } \\
\text { Mattingly, Talwalkar et al. 2006; } \\
\text { Bai, Wei et al. 2010; Kim, Pyun et } \\
\text { al. 2010) }\end{array}$ \\
\hline
\end{tabular}

\subsection{Finite Element Solver and Mesh Properties}

3.7. Out of the common solvers used in the finite element domain, ABAQUS (Martins, Pato et al. 2007; Phillips, Pankaj et al. 2007; Tang, Chan et al. 2010) and LS-DYNA (Anderson, Peters et al. 2004; Silvestri and Ray 2009; Bai, Wei et al. 2010) were used in three studies each, while ANSYS was used in two studies (Wagnac, Aubin et al. 2008; El-Asfoury and El-Hadek 2009). Other less common solvers such as TrueGrid 1.4 (Camacho, Ledoux et al. 2002) and $3 D$ ViewNX (Mattingly, Talwalkar et al. 2006) were used each in one study. The rest of studies did not specify a solver for their FEM. A summary of the mesh properties for all the studies is presented in CASP Appraisal Results

Each of the selected studies was screened against the eleven questions forming the CASP method. The results of the screening are outlined in Table 3. From these results, the average CASP score was $7 / 11$ with the lowest score of $3 / 11$ and the highest score being 10/11. The standard deviation was 1.96 .

\section{Discussion}

After analysing the results outlined in 
Table 1, it can be stated that most of the attention is dedicated to developing models of the pelvis, the buttocks and the femur, whereas other segments such as the shank and the ankle lack attention in the domain of finite element modeling. This suggests that researchers need to scale efforts in order to attain a balance and fill-in the gaps present in the human body FEM domain.

From the CASP appraisal (Table 3), it can be seen that the average score for all the studies is $7 / 11$, which indicates an acceptable level of relevance and quality. However, the results in Table 3 include a few outliers, which indicate a significant spread within the scores. Furthermore, from the same table, eight studies out of fifteen were able to achieve reasonable validation for their models, which indicates an acceptable level of reliability of the models. On the other hand, inspection of results of the literature review indicated a notable bias in modeling males compared to females. Out of the eight studies, which specified a gender and percentile for their models, only two modeled a female. Furthermore, out of the same eight models, four developed models to represent the $50^{\text {th }}$ percentile male and one study developed a subjectspecific model based on males who could closely resemble a $50^{\text {th }}$ percentile male in stature.

In terms of methodological bias or quality, Table 3 indicates that only five out of fifteen studies strived to avoid bias in the results. For example, the difference between the male and the female bodies is a potential source of bias. Having only five studies taking action to reduce the effects of such possible bias can produce a notable variability within the overall CASP scores. Similarly, only five studies accounted for confounding factors, such as variation of loading conditions and its influence on posture, or the validity of using supine MRI data to model a sitting human's body. This further contributes to the wide variability within the overall CASP scores.

Finally, when assessing the data used to create finite element models of the human lower body, an inference can be made by relating Table 3 with the data sources of the studies (section 3.2). It can be stated with confidence that Visible Human Project data can be used as a source of data for finite element modeling of the human lower body. Similarly, SizeUSA can be considered a valid database for finite element modeling of the human lower body, however, with less confidence than the Visible Human Project data. This is mainly because this review was able to indentify only one study which used SizeUSA as their data source and validated their model, whereas three studies were identified to base their models on the Visible Human Project data and all three provided reasonable validation of their produced models. On the other hand, no conclusion can be drawn whether other databases can be considered as a valid data source for building finite element human lower body models, as no recent study with reasonable validation was identified to have used other databases as their source of data.

\section{Conclusions}

In this study, a literature review was conducted to identify recent finite element models of the human pelvis and lower extremity. Fifteen studies were identified to be of relevance and match the inclusion criteria, each of which was screened against modified CASP appraisal criteria. The appraisal of literature indicated that the literature listed in this review was of acceptable relevance and quality. On the other hand, the findings of the literature review indicate that there is a bias towards modeling males rather than females. Furthermore, within malepopulation modeling, the literature review shows a tendency toward modeling $50^{\text {th }}$ percentile males compared to other percentiles of the population. Moreover, an unbalance in modeling different body segments was noted as the number of models for the pelvis significantly exceeded those of the ankle or the shank. Additionally, findings of the literature 
review indicate that the Visible Human project data can be used to develop a finite element of the lower extremity and the pelvis of the human with an acceptable level of confidence compared to other databases.

Table 2.

\subsection{CASP Appraisal Results}

Each of the selected studies was screened against the eleven questions forming the CASP method. The results of the screening are outlined in Table 3 . From these results, the average CASP score was $7 / 11$ with the lowest score of $3 / 11$ and the highest score being 10/11. The standard deviation was 1.96 .

\section{Discussion}

After analysing the results outlined in

Table 1, it can be stated that most of the attention is dedicated to developing models of the pelvis, the buttocks and the femur, whereas other segments such as the shank and the ankle lack attention in the domain of finite element modeling. This suggests that researchers need to scale efforts in order to attain a balance and fill-in the gaps present in the human body FEM domain.

From the CASP appraisal (Table 3), it can be seen that the average score for all the studies is $7 / 11$, which indicates an acceptable level of relevance and quality. However, the results in Table 3 include a few outliers, which indicate a significant spread within the scores. Furthermore, from the same table, eight studies out of fifteen were able to achieve reasonable validation for their models, which indicates an acceptable level of reliability of the models. On the other hand, inspection of results of the literature review indicated a notable bias in modeling males compared to females. Out of the eight studies, which specified a gender and percentile for their models, only two modeled a female. Furthermore, out of the same eight models, four developed models to represent the $50^{\text {th }}$ percentile male and one study developed a subjectspecific model based on males who could closely resemble a $50^{\text {th }}$ percentile male in stature.

In terms of methodological bias or quality, Table 3 indicates that only five out of fifteen studies strived to avoid bias in the results. For example, the difference between the male and the female bodies is a potential source of bias. Having only five studies taking action to reduce the effects of such possible bias can produce a notable variability within the overall CASP scores. Similarly, only five studies accounted for confounding factors, such as variation of loading conditions and its influence on posture, or the validity of using supine MRI data to model a sitting human's body. This further contributes to the wide variability within the overall CASP scores.

Finally, when assessing the data used to create finite element models of the human lower body, an inference can be made by relating Table 3 with the data sources of the studies (section 3.2). It can be stated with confidence that Visible Human Project data can be used as a source of data for finite element modeling of the human lower body. Similarly, SizeUSA can be considered a valid database for finite element modeling of the human lower body, however, with less confidence than the Visible Human Project data. This is mainly because this review was able to indentify only one study which used SizeUSA as their data source and validated their model, whereas three studies were identified to base their models on the Visible Human Project data and all three provided reasonable validation of their produced models. On the other hand, no conclusion can be drawn whether other databases can be considered as a valid data source for building finite element human lower body 
models, as no recent study with reasonable validation was identified to have used other databases as their source of data.

\section{Conclusions}

In this study, a literature review was conducted to identify recent finite element models of the human pelvis and lower extremity. Fifteen studies were identified to be of relevance and match the inclusion criteria, each of which was screened against modified CASP appraisal criteria. The appraisal of literature indicated that the literature listed in this review was of acceptable relevance and quality. On the other hand, the findings of the literature review indicate that there is a bias towards modeling males rather than females. Furthermore, within malepopulation modeling, the literature review shows a tendency toward modeling $50^{\text {th }}$ percentile males compared to other percentiles of the population. Moreover, an unbalance in modeling different body segments was noted as the number of models for the pelvis significantly exceeded those of the ankle or the shank. Additionally, findings of the literature review indicate that the Visible Human project data can be used to develop a finite element of the lower extremity and the pelvis of the human with an acceptable level of confidence compared to other databases.

Table 2: Studies addressed in the literature review and the corresponding mesh properties.

\begin{tabular}{|l|l|}
\hline Study & Mesh properties \\
\hline 2005) & $\begin{array}{l}\text { Femur: 220 shell } \\
\text { elements } \\
\text { Tibia: 176 shell elements }\end{array}$ \\
\hline (Silvestri and Ray 2009) & $\begin{array}{l}\text { 28856 solid elements } \\
8468 \text { shell elements } \\
150 \text { discrete elements }\end{array}$ \\
\hline $\begin{array}{l}\text { (Camacho, Ledoux et al. } \\
\text { 2002) } \\
\text { elements four-noded shell } \\
2112 \\
\text { hexagonal elements }\end{array}$ \\
\hline (Martins, Pato et al. \\
2007) & $\begin{array}{l}\text { Three-noded and four- } \\
\text { noded elements * }\end{array}$ \\
\hline (Shim, Pitto et al. 2007) & $\begin{array}{l}\text { Eight-noded hexagonal } \\
\text { and six-noded pentagonal } \\
\text { elements * }\end{array}$ \\
\hline (El-Asfoury and El- & $\begin{array}{l}450168 \text { nodes } \\
\text { 343690 elements }\end{array}$ \\
Hadek 2009) & 2D strain elements * \\
\hline (Tang, Chan et al. 2010) & Four-noded tetrahedral \\
\hline (Phillips, Pankaj et al.
\end{tabular}

\begin{tabular}{|l|l|}
\hline 2007) & $\begin{array}{l}\text { elements, six-noded } \\
\text { linear elements, solid } \\
\text { shells and wedge shaped } \\
\text { elements * }\end{array}$ \\
\hline $\begin{array}{l}\text { (Wagnac, Aubin et al. } \\
\text { 2008) }\end{array}$ & $\begin{array}{l}\text { Buttocks: 29292 four- } \\
\text { noded tetrahedral } \\
\text { elements } \\
\text { Cushion: 846 eight-noded } \\
\text { brick elements }\end{array}$ \\
\hline $\begin{array}{l}\text { (Kim, Pyun et al. 2010) } \\
\text { Tetragonal solid elements }\end{array}$ \\
2004) & $\begin{array}{l}\text { Four-noded, 24 degree of } \\
\text { freedom elements * }\end{array}$ \\
\hline $\begin{array}{l}\text { (Cheng, Smith et al. } \\
\text { 2007), (Mattingly, } \\
\text { Talwalkar et al. 2006), } \\
\text { (Bai, Wei et al. 2010), } \\
\text { (Makhsous and Lin } \\
\text { 2009) }\end{array}$ & *NUMBER OF ELEMENTS NOT MENTIONED \\
AELMENT TYPES NOT MENTIONED \\
!MESH PROPERTIES NOT MENTIONED
\end{tabular}


Table 3: CASP (modified LOW appraisal criteria) analysis for all studies addressed in the literature review

\begin{tabular}{|c|c|c|c|c|c|c|c|c|c|c|c|c|c|c|c|}
\hline Questions & 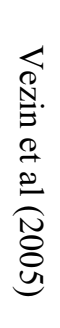 & 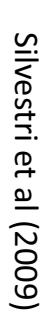 & 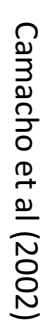 & 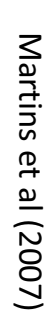 & 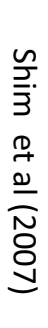 & 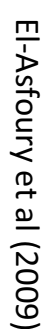 & 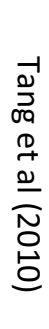 & 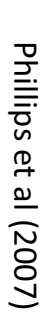 & 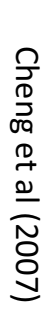 & 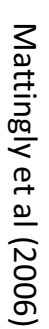 & 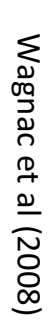 & 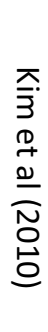 & 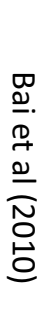 & 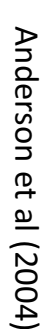 & 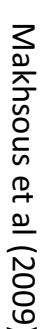 \\
\hline $\begin{array}{l}\text { Did the study address a } \\
\text { clearly focused issue? }\end{array}$ & $Y$ & $Y$ & $Y$ & $Y$ & $Y$ & $Y$ & $Y$ & $Y$ & $Y$ & $Y$ & $Y$ & $Y$ & $Y$ & $Y$ & $Y$ \\
\hline $\begin{array}{l}\text { Did the authors use an } \\
\text { appropriate method to } \\
\text { answer their questions? }\end{array}$ & $\mathrm{Y}$ & $Y$ & $\mathrm{Y}$ & $Y$ & $Y$ & $Y$ & $Y$ & $Y$ & $Y$ & $Y$ & $Y$ & $Y$ & $Y$ & $Y$ & $Y$ \\
\hline $\begin{array}{c}\text { Was the data collected } \\
\text { from a clear and suitable } \\
\text { source? }\end{array}$ & $Y$ & $Y$ & $?$ & $Y$ & $\mathrm{Y}$ & $Y$ & $?$ & $\mathrm{Y}$ & $Y$ & $Y$ & $Y$ & $Y$ & $Y$ & $Y$ & Y \\
\hline $\begin{array}{c}\text { Were the controls } \\
\text { selected in an acceptable } \\
\text { way? }\end{array}$ & $Y$ & $Y$ & $Y$ & $Y$ & $Y$ & $?$ & $Y$ & $Y$ & $Y$ & $Y$ & $Y$ & $Y$ & ? & $Y$ & $Y$ \\
\hline $\begin{array}{l}\text { Did the authors dedicate } \\
\text { a means to avoid bias? }\end{array}$ & $Y$ & $\mathrm{~N}$ & $Y$ & $\mathrm{~N}$ & $\mathrm{~N}$ & $\mathrm{~N}$ & $\mathrm{~N}$ & $\mathrm{~N}$ & $Y$ & $Y$ & $\mathrm{~N}$ & $Y$ & $\mathrm{~N}$ & $\mathrm{~N}$ & $?$ \\
\hline $\begin{array}{l}\text { Have the authors taken } \\
\text { account of the potential } \\
\text { confounding factors in } \\
\text { the design and/or in } \\
\text { their analysis? }\end{array}$ & $?$ & $?$ & $Y$ & $\mathrm{~N}$ & $Y$ & $\mathrm{~N}$ & $\mathrm{~N}$ & $?$ & $N$ & $?$ & $\mathrm{~N}$ & $Y$ & $?$ & $Y$ & $Y$ \\
\hline $\begin{array}{c}\text { Are the results clear to } \\
\text { the reader? }\end{array}$ & $\mathrm{N}$ & $Y$ & $Y$ & $\mathrm{Y}$ & $Y$ & $Y$ & $Y$ & $Y$ & $Y$ & $Y$ & $Y$ & $Y$ & $\mathrm{~N}$ & $Y$ & $Y$ \\
\hline Are the results precise? & $?$ & $?$ & $?$ & $?$ & $\mathrm{~N}$ & $?$ & $?$ & $?$ & $?$ & $\mathrm{Y}$ & $\mathrm{Y}$ & $\mathrm{N}$ & $?$ & $\mathrm{Y}$ & $Y$ \\
\hline Is the model validated? & $\mathrm{Y}$ & $\mathrm{Y}$ & $\mathrm{N}$ & $\mathrm{N}$ & $\mathrm{Y}$ & $\mathrm{N}$ & $\mathrm{N}$ & $\mathrm{Y}$ & $\mathrm{N}$ & $\mathrm{N}$ & $\mathrm{Y}$ & $\mathrm{Y}$ & $\mathrm{N}$ & $\mathrm{Y}$ & $\mathrm{Y}$ \\
\hline $\begin{array}{l}\text { Is the model applicable } \\
\text { to a general population? }\end{array}$ & $\mathrm{Y}$ & $\mathrm{Y}$ & ? & $\mathrm{N}$ & $?$ & $\mathrm{~N}$ & ? & $\mathrm{N}$ & $Y$ & $?$ & $\mathrm{~N}$ & $Y$ & $\mathrm{~N}$ & $\mathrm{~N}$ & $?$ \\
\hline $\begin{array}{c}\text { Do the results fit with } \\
\text { other available } \\
\text { evidence? }\end{array}$ & $?$ & $Y$ & $Y$ & $?$ & $Y$ & $?$ & $\mathrm{Y}$ & $Y$ & $?$ & $?$ & $Y$ & $Y$ & $?$ & $Y$ & $Y$ \\
\hline Score out of 11 & 7 & 8 & 8 & 5 & 8 & 4 & 5 & 7 & 7 & 7 & 8 & 10 & 3 & 9 & 9 \\
\hline
\end{tabular}

$\mathrm{Y}=\mathrm{Yes}$

$\mathrm{N}=\mathrm{No}_{\mathrm{o}}$

$?=$ Can't tell 


\section{References}

Amann, C., A. Huschenbeth, et al. (2009). "Virtual Assessment of Occupied Seat Vibration Transmissibility." SAE International Journal of Passenger Cars-Electronic and Electrical Systems 1(1): 574-579.

Amann, C., T. Klisch, et al. (2005). Virtual seat comfort assessment. VDI Congress. Braunschweig.

Anderson, A. E., C. L. Peters, et al. (2004). "A Subject Specific Finite Element Model of the Pelvis: Development, Validation and Sensitivity Studies." Journal of Biomechancal Engineering.

Bai, X., G. Wei, et al. (2010). "Finite Element Musculloskeletal Modeling of Mechanical Virtual Human of China." 1.

Camacho, D. L. A., W. R. Ledoux, et al. (2002). "A Three-Dimensional, Anatomically Detailed Foot Model: A Foundation for a Finite Element Simulation and Means of Quantifying Foot-Bone Position." Journal of Rehabilitaion Research and Develpoment 39(3): 401 - 410.

Cheng, Z., J. A. Smith, et al. (2007). "Considerations and Experiences in Developing a Finite Element Buttock Model for Seating Comfort Analysis." 1(2458).

Chow, W. W. and E. I. Odell (1978). "Deformation and Stress in Soft Body Tissue of a Sitting Person." Journal of Biomechancal Engineering: 79-87.

El-Asfoury, M. S. and M. A. El-Hadek (2009). "Static and Dynamic Three-Dimensional Finite Element Analysis of Pelvic Bone." International Journal of Mathermatical, Physical and Engineering Sciences 3(1).

ESI (2010). "PAM-COMFORT ". Retrieved May 2010, from http://www.esigroup.com/products/comfort/pam-comfort.

Guyatt, G. H., D. Sackett, et al. (1993). Users' Guide to the Medical Literature II: How to Use an Article About Therapy or Prevention.

Kim, S. H., J. K. Pyun, et al. (2010). "Digital Human Body Model for Seat Comfort Simulation."
International Journal of Automotive Technology: $239-244$.

Makhsous, M. and F. Lin (2009). "A Finite-Element Biomechanical Model for Evaluation Buttock Tissue Loads in Seated Individuals with Spinal Cord Injury." Bioengineering Research of Chronic Wounds 1: 181-205.

Martins, J. A. C., M. P. M. Pato, et al. (2007). "Finite Element Studies of the Deformation of the Pelvic Floor." New York Academy of Sciences: 316 - 334.

Mattingly, B., V. Talwalkar, et al. (2006). "ThreeDimensional in Vivo Motion of Adult Hind Foot Bones." Journal of Biomechanics 39: 726 - 733.

McConville, J., C. Clauser, et al. (1980). Anthropometric relationships of body and body segment moments of inertia. Wright-Patterson Air Force Base. Ohio.

Pankoke, S. and A. Siefert (2007). Virtual Simulation of Static and Dynamic Seating Comfort in the Development Process of Automobiles and Automotive Seats: Application of the FiniteElement-Occupant-Model CASIMIR. 2007 Digital Human Modeling Conference. Washington.

Phillips, A. T. M., P. Pankaj, et al. (2007). "Finite Element Modelling of the Pelvis: Inclusion of Muscular and Ligamentous Boundary Conditions." Medical Engineering and Physics: 739 -748.

Shim, V. B., R. P. Pitto, et al. (2007). "The Use of Sparse CT Datasets for Auto-Generating Accurate FE Models of the Femur and Pelvis." Journal of Biomechanics 40: 26-35.

Silvestri, C. and M. H. Ray (2009). "Development of a Finite Element Model of the Knee-Thigh-Hip of a 50th Percentile Male Including Ligaments and Muscles." International Journal of Crashworthiness 14(2): 215 - 229 .

Tang, C. Y., W. Chan, et al. (2010). "Finite Element Analysis of Contact Pressure Between Seat Cushion and Human Buttock-Thigh Tissue." $\underline{\text { Scientific }}$ Research (Engineering): 720 - 726. 
Vezin, P. and J. P. Verriest (2005). "Development of a Set of Numerical Human Models for Safety ".

Wagnac, E. L., C.-E. Aubin, et al. (2008). "A New Method to Generate a Patient-Specific Finite Element Model of the Human Buttocks." IEEE Transaction on Biomedical Engineering 55(2): 774 783.

VHP2011.http:/www.nlm.nih.gov/research/visible/ visible_human.html. Visited on 14Mar 2011
CVHP 2011,

http://www.chinesevisiblehuman.com/index_e.aspx. Visited 14Mar2011

VAKHUM 2011.

http://www.biomedtown.org/biomed_town/LHDL/R eception/datarepository/repositories/VAKHUM_ho me. Visited on 14Mar 2011.

SizeUSA 2011. http://www.tc2.com/sizeusa.html. Visited on 14March 2011 